\title{
Transcending marine turtles: first report of hatching failure in eggs of Amazonian freshwater turtles with symptoms of the fungal emerging disease fusariosis
}

\author{
Ana Sofía Carranco ${ }^{1}$, Mark A.F. Gillingham ${ }^{1}$, Kerstin Wilhelm ${ }^{1}$, María de Lourdes \\ Torres $^{2}$, Simone Sommer ${ }^{1}$, and David Romo ${ }^{2}$ \\ ${ }^{1}$ Universitat Ulm \\ ${ }^{2}$ Universidad San Francisco de Quito
}

November 3, 2021

\begin{abstract}
In the last decades fungal pathogens are causing devastating population declines across a broad range of taxa. A newly emerging fungal disease, sea turtle egg fusariosis, caused by members of the Fusarium solani species complex (FSSC), has been reported to be responsible for hatching failure in sea turtles around the world. However, this has not been reported in other non-marine turtle species. Herein we report high hatching failure from eggs symptomatic of fusariosis in the yellow-spotted Amazon river turtle ( Podocnemis unifilis), inhabiting a pristine environment in the Ecuadorian Amazon. We assessed hatching success from eggs symptomatic and asymptomatic of fusariosis ( $n=680$ eggs), tested for Fusarium infection by PCR amplifying the TEF-1 $\alpha$ gene $(n=68$ turtle internal egg swab samples) and sequenced eight amplicons for screening of FSSC membership on an Illumina Miseq. Hatchability was $72 \%$ for asymptomatic eggs, whilst only $8 \%$ of symptomatic eggs hatched. Eight percent of asymptomatic and $58 \%$ of symptomatic eggs tested positive for Fusarium spp. and sequencing revealed that nine sequence variants from three asymptomatic and four symptomatic eggs corresponded to F. keratoplasticum, F. solani and F. falciforme, the three major FSSC pathogens already reported in sea turtle egg fusariosis. Our study therefore suggests that observed hatching failure of eggs showing symptoms of fusariosis is at least partially caused by Fusarium pathogens within FSSC in a freshwater turtle. This report highlights that fusariosis is more widespread among the Testudines order than previously reported and is not limited to sea environments, which is of particular conservation concern.
\end{abstract}

\section{Introduction}

In the last two decades there has been an unprecedented and worldwide emergence of fungal pathogens threatening animal and plant biodiversity (Fisher et al. 2012). Recently, there has been increasing concern over the emerging fungal pathogen Fusarium in endangered sea turtles, which causes hatching failure in eggs of sea turtles worldwide (Smyth et al., 2019). So far, reports of the pathogenesis and distribution of this pathogen has been limited to sea turtles, and it is unknown whether this pathogen also poses a threat to freshwater and terrestrial turtle species, of which there are 356 known species (Rhodin et al., 2017).

First reports of sea turtle eggs colonized by fungi were done by Wynecken et al. (1988), who described hatching failure of diseased eggs within natural and artificial nests. Since then, several studies have reported the presence of fungi (Phillott \& Parmenter, 2001) and bacteria (Craven et al., 2007) in unhatched eggs and female cloaca, indicating that turtle eggs are not laid sterile and have a commensal surface microbiome. Fungi from the genus Fusarium were identified as pathogenic to sea turtle eggs (Sarmiento-Ramírez et al., 2010), and in 2012 Fusarium solani was recognized as a new fungal emerging infectious disease (EID) that may be contributing to population declines in sea turtles (Fisher et al., 2012). Infections caused by 60 species belonging to the Fusarium solani species complex (FSSC) have been reported in sea turtles around 
the globe (Brofft Bailey et al., 2018; Candan, 2018; Sarmiento-Ramírez et al., 2014).Fusarium has been isolated from both diseased and asymptomatic sea turtle eggs, and infection may be vertically transmitted from the mother cloaca or horizontally transmitted from the environment (Sarmiento-Ramírez et al., 2014). Given the large number of Fusarium species and their global range, it is possible that this fungal disease is not limited to sea environments and may also be spreading to freshwater environments. Indeed, Fusarium is thought to thrive in water and damp environments (Smyth et al., 2019), and therefore fresh-water turtle species may also be at risk, yet there has been to date little surveillance of non-oceanic turtle species.

In this study, we screened 680 eggs from artificial nests of an endangered freshwater turtle species $(P$. unifilis ) that inhabits the Amazon and Orinoco basin in South America for visual symptoms of fusariosis and assessed hatchability. We tested 68 eggs by PCR for Fusarium infection and sequenced eight of those amplicons on an Illumina platform to screen for members of FSSC.

\section{Materials and Methods}

\section{Study site and sample collection}

Sampling and data collection were performed from March to May 2019 and April to May 2020 in artificial nests relocated at the Tiputini Biodiversity Station (TBS) (037'05"S, 7610'19"W, 190-270 m a.s.l) situated in the Yasuni Biosphere Reserve in the Orellana province of Ecuador. After the incubation period (3 months) we determined symptoms of fungal infections by visual inspection. Eggs were cataloged as symptomatic eggs when covered with unusual colored spots (green, pink, greyish) and with a non-uniform shape (Figure 1a), and eggs without these traces were cataloged as asymptomatic. In 2019, we sampled inner eggshells of 68 eggs (29 asymptomatic, 39 symptomatic) using a sterile swab. In 2020, hatchability data of 680 eggs (394 asymptomatic eggs, 286 symptomatic) from 23 different nests was collected. See supplementary information for detailed information on sampling design and data collection.

\section{DNA extraction and Sequencing}

DNA was extracted from 68 inner-eggshell swabs using the NucleoSpin@ 96 Soil extraction kit (MachereyNagel, Germany) following kit instructions. The PCR amplification of the TEF-1 $\alpha$ gene was performed using Fusarium -specific primers with the cycling conditions reported previously (Cobo-Díaz et al., 2019). Eight inner-eggshell swabs (four-symptomatic and four-asymptomatic eggs) were sequenced following the Illumina MiSeq sequencing methodology as described by Menke et al. (2017). More detailed information is provided in supplementary material.

\section{Bioinformatics and phylogenetic analysis}

Paired reads from Illumina MiSeq sequencing were processed with the open-source QIIME2 software (version 2019.1) (Bolyen et al., 2018) and the DADA2 pipeline (Callahan et al., 2016) to denoise the dataset from artefacts and to generate amplicon sequence variants (ASVs). We assigned taxonomy within QIIME2 by building a classifier using the RESCRIPt (Robeson et al., 2020) and the NCBI database using BLASTnt (Table S1). For phylogenetic analyses we included Fusarium spp., isolated from sea turtles' eggs, crops, environment and humans, and in addition, we includedFusarium spp., from the Fusarium Tricinctum Species Complex (FTSC) and Fusarium Fujikuroi Species Complex (FFSC), to show the different complexes' clusters. We aligned and generated a Bayesian phylogenetic tree with 33 sequences and an outgroup sequence by using the MrBayes 3.2.6 (Ronquist et al., 2012) module within Geneious v.11.0.5 (Kearse et al., 2012). Detailed information on bioinformatics and the phylogenetic analysis is provided in the supplementary material.

\section{Statistical analyses}

To investigate whether infection status varied across nests we used a General Linear Model (GLM) with a binomial distribution within the R statistical software (RStudio Team; 2020). To investigate hatching success (hereafter hatchability) according to fusariosis symptom status, we performed a Generalized Linear Mixed Model (GLMM) with a binomial distribution using the R package "lme4" (Bates et al., 2015). To control for pseudo-replication, we entered nest ID as a random factor. 


\section{Results and Discussion}

Several studies have reported and confirmed infections by Fusarium pathogens in sea turtles around the world and have investigated the relationship between FSSC infections and hatching success (e.g. Sarmiento-Ramirez et al., 2010; 2014). However, the negative effects of FSSC in freshwater turtles have not been reported until now. We found that all 23 nests of the yellow-spotted turtle monitored in this study had eggs showing symptoms of fusariosis, with an average of $42 \%$ of eggs symptomatic across nests (range 10-100\%; Table S2). There was significant variation in the proportion of diseased eggs between nests $\left(\mathrm{X}^{2}{ }_{1,22}=192.48\right.$; $\mathrm{p}$-value $<$ $\left.0.001 ; \operatorname{adj}-\mathrm{R}^{2}=0.332\right)$ suggesting that some nests were more vulnerable to infection than others. Symptoms of FSSC infections in eggs of the yellow-spotted turtle were significantly associated with hatching success $\left(\mathrm{X}^{2}{ }_{1,677}=149.86 ; \mathrm{p}\right.$-value $<0.001 ;$ adj- $\left.\mathrm{R}^{2}=0.525\right)$ with a $72 \%$ hatching success for asymptomatic eggs $(n=394)$, whilst only $8 \%$ of symptomatic eggs hatched $(n=286)$ (Figure $1 \mathrm{~b})$. In line with our results, experimental inoculation of $F$. solani in eggs of the sea turtleCaretta caretta resulted in a hatching success rate of $18 \%(n=12)$ compared to $92 \%(n=12)$ for uninfected controls (Sarmiento-Ramirez et al., 2010). Similar disparity in hatching rates according to symptoms of fusariosis were also observed in the natural environment in endangered sea turtles, with up to $92 \%$ embryo mortality in diseased eggs (Sarmiento-Ramirez et al., 2014).

Fusarium -specific PCR tests of 29 asymptomatic and 39 symptomatic eggs revealed that $59 \%$ of the symptomatic eggs tested positive forFusarium (Table S3), whilst the remaining $41 \%$ tested negative (Table S3), suggesting that infected eggs may not only be afflicted by pathogenic Fusarium and that there are multiple causes of infection. Indeed, previous studies have reported infections of sea-turtle eggs caused by different fungi within Aspergillus, Rhizopus and Apophysomyces (Candan, 2018; Santos Costa-Neves et al., 2015). Interestingly, we also found that $28 \%$ of asymptomatic eggs tested positive for Fusarium (Table S3), suggesting either that these eggs were at an initial stage of infection or that colonization of Fusarium did not lead to the onset of pathogenic fusariosis. Indeed, although the pathogenicity of $F$. solani in sea turtles has been demonstrated, it is not well understood if colonization is systematically pathogenic and other species of Fusarium may not be pathogenic (Sarmiento-Ramirez et al., 2010).

From our sequencing of amplicons (from three asymptomatic and four symptomatic eggs), we obtained nine Fusarium amplicon sequence variants (ASVs) belonging to the pathogenic FSSC ( $\mathrm{PP}=1$; Figure 2). From the obtained ASVs, ASV_1 and ASV_3 were present in both asymptomatic and symptomatic eggs, ASV_4 and ASV_5 were each present in two different symptomatic eggs, and the remaining five ASVs were only present in asymptomatic eggs (Table S4). ASV_3 and ASV_6 grouped withF. keratoplasticum and F.solani isolates from human, crops and sea turtle egg infections. ASV_4 grouped with $F$. solaniisolated from crops and $P$. unifilis eggs $(\mathrm{PP}=0.98$; Figure 2$)$. ASV $\_2,-5$, and $\_7$ grouped with $F$. falciforme isolates from the environment, crops, human, and sea turtle egg infections ( $\mathrm{PP}=0.915$; Figure 2). In contrast, ASV_1, _8 and -9 grouped together with $\operatorname{six} F$. keratoplasticum isolates from $P$. unifilis eggs $(\mathrm{PP}=0.933$; Figure 2). Our results add novel data to a recent study reporting the presence of $F$. keratoplasticum in $P$. unilfilis eggs in artificial nests of the same geographical region (García-Martín et al., 2021) by adding the incidence of the FSSC's F. falciforme andF.solani .

Although both our study and that of García-Martín et al. (2021) were conducted on harvested eggs, the source of pathogenic FSSC is likely to be vertical transmission from the mother or horizontal transmission from the natural environment (since eggs are incubated in sand from the original nesting site). Whilst it is possible that infection may spread more easily within artificial nests than in natural settings, our combined results suggest a possible Fusarium infection outbreak in a remote area of the Amazon and that it is likely to be a major contributor of infectious hatching failure in this freshwater turtle species. This has important implications for turtle conservation efforts worldwide, since egg harvesting, and artificial incubation is a major strategy applied by turtle conservationists. Further studies are necessary to understand the epidemiology and distribution of Fusarium pathogenic fungi causing hatching failure of endangered non-marine turtle species worldwide.

\section{Acknowledgments}


This study was done under the collection research-permit No.MAE-DNB-CM-2016-0046-M-0003 and exportation permit No.158-2019-EXP-CM-FAU-DNB/MA awarded by the Environmental Ministry of Ecuador. Many thanks to the TBS and its staff, to Gabriela Vinueza and Diego Mosquera involved in the P. unifilis project helping with data collection, to Tomi Sugahara for assistance on collection permits, Ulrike Stehle for assistance in laboratory work and Alice Risely for comments and feedback. This project was funded by the Institute of Evolutionary Ecology and Conservation Genomics of the Ulm University and the TBS.

\section{Conflict of Interest Statement}

The authors declare no conflict of interests regarding this study.

\section{Author contribution}

S.S., M.G. and A.S.C. conceived the idea; all authors contributed to the study design; D.R., M.L.T. and A.S.C. led sampling and field work logistics. A.S.C. collected the data; A.S.C. and M.G. led the analysis; A.S.C. and M.G. led the manuscript writing. All authors contributed critically to the drafts and gave final approval for publication.

\section{Data availability statement}

Individual Fusarium TEF 1-alpha gene raw-sequences are available in the NCBI Sequence Read Archive (SRA) under BioProject PRJNA745100 with accession number SAMN20145776.

\section{References}

Bates D, Mächler M, Bolker B, Walker S (2015). Fitting linear mixed-effects models

using lme4. Journal of Statistical Software, 67(1), 1-48. doi: 10.18637/jss.v067.i01.

Bolyen, E., Rideout, J. R., Dillon, M. R.,... Caporaso, J. G. (2018). QIIME 2: Reproducible, interactive, scalable, and extensible microbiome data science [Preprint]. PeerJ Preprints. https://doi.org/10.7287/peerj.preprints.27295v2

Brofft B., J., Lamb, M., Walker, M., Weed, C., \& Stephenson C., K. (2018). Detection of potential fungal pathogens Fusarium falciforme and F. keratoplasticum in unhatched loggerhead turtle eggs using a molecular approach. Endangered Species Research, 36, 111-119. https://doi.org/10.3354/esr00895

Callahan, B. J., McMurdie, P. J., Rosen, M. J., ... Holmes, S. P. (2016). DADA2: High-resolution sample inference from Illumina amplicon data.Nature Methods, 13(7), 581-583. https://doi.org/10.1038/nmeth.3869

Candan, E. D. (2018). Molecular identification of fungal isolates and hatching success of green turtle (Chelonia mydas) nests. Archives of Microbiology, 200(6), 911-919. https://doi.org/10.1007/s00203-018-1496-0

Cobo-Díaz, J. F., Baroncelli, R., Le Floch, G., \& Picot, A. (2019). A novel metabarcoding approach to investigate Fusarium species composition in soil and plant samples. FEMS Microbiology Ecology, 95(7), fiz084. https://doi.org/10.1093/femsec/fiz084

Craven, K., Awong-Taylor, J., Griffiths, L., Bass, C., \& Muscarella, M. (2007). Identification of bacterial isolates from unhatched loggerhead (Caretta caretta) sea turtle eggs in Georgia, USA. Marine Turtle Newsletter $, 115,9-11$.

Fisher, M. C., Henk, Daniel. A., Briggs, C. J., Brownstein, J. S., Madoff, L. C., McCraw, S. L., \& Gurr, S. J. (2012). Emerging fungal threats to animal, plant and ecosystem health. Nature, 484(7393), 186-194. https://doi.org/10.1038/nature10947

García-Martín, J. M., Sarmiento-Ramírez, J. M., \& Diéguez-Uribeondo, J. (2021). Beyond sea turtles: Fusarium keratoplasticum in eggshells of Podocnemis unifilis, a threatened Amazonian freshwater turtle. Journal of Fungi , 7 (9), 742. https://doi.org/10.3390/jof7090742 
Kearse, M., Moir, R., Wilson, A., Stones-Havas, S., Cheung, M., Sturrock, S., Buxton, S., Cooper, A., Markowitz, S., Duran, C., Thierer, T., Ashton, B., Meintjes, P., \& Drummond, A. (2012). Geneious Basic: An integrated and extendable desktop software platform for the organization and analysis of sequence data. Bioinformatics , 28 (12), 1647-1649. https://doi.org/10.1093/bioinformatics/bts199

Menke, S., Gillingham, M. A. F., Wilhelm, K., \& Sommer, S. (2017). Home-made cost effective preservation buffer is a better alternative to commercial preservation methods for microbiome research. Frontiers in Microbiology,8. https://doi.org/10.3389/fmicb.2017.00102

Phillott, Andrea D., \& Parmenter, C. J. (2001). The distribution of failed eggs and the appearance of fungi in artificial nests of green (Chelonia mydas) and loggerhead (Caretta caretta) sea turtles. Australian Journal of Zoology, 49(6), 713. https://doi.org/10.1071/ZO00051

Rhodin, A. G. J., Iverson, J. B., Bour, R., Fritz, U., Georges, A., Shaffer, H. B., \& van Dijk, P. P. (2017). Turtles of the world: Annotated checklist and atlas of taxonomy, synonymy, distribution, and conservation status (8th Ed.). Chelonian Research Foundation \& Turtle Conservancy. https://doi.org/10.3854/crm.7.checklist.atlas.v8.2017

Robeson, M. S., O'Rourke, D. R., Kaehler, B. D., Ziemski, M., Dillon, M. R., Foster, J. T., \& Bokulich, N. A. (2020). RESCRIPt: Reproducible sequence taxonomy reference database management for the masses [Preprint]. Bioinformatics. https://doi.org/10.1101/2020.10.05.326504

Ronquist, F., Teslenko, M., van der Mark, P., Ayres, D. L., Darling, A., Höhna, S., ... Huelsenbeck, J. P. (2012). MrBayes 3.2: Efficient bayesian phylogenetic inference and model choice across a large model space.Systematic Biology, 61(3), 539-542. https://doi.org/10.1093/sysbio/sys029

RStudio Team. (2020). RStudio: Integrated development environment for R (Version 3.6.3). Boston, MA: RStudio, Inc. Retrieved from http://www.rstudio.com/

Santos Costa Neves, M., Carneiro de Melo Moura, C., \& Goncalves de Oliveira, L. (2015). Mycobiota from the eggs, nests and stillbirths of Eretmochelys imbricata Linneus 1766 (Testudines: Cheloniidae) in Pernambuco State, Brazil. African Journal of Microbiology Research, 9(17), 1195-1199. https://doi.org/10.5897/AJMR2015.7389

Sarmiento-Ramírez, J. M., Abella, E., Martín, M. P., Tellería, M. T., López-Jurado, L. F., Marco, A., \& Diéguez-Uribeondo, J. (2010). Fusarium solani is responsible for mass mortalities in nests of loggerhead sea turtle, Caretta caretta, in Boavista, Cape Verde: Fusarium solaniin sea turtle. FEMS Microbiology Letters, 312(2), 192-200. https://doi.org/10.1111/j.1574-6968.2010.02116.x

Sarmiento-Ramírez, J. M., Abella-Pérez, E., Phillott, A. D., Sim, J., van West, P., Martín, M. P., Marco, A., \& Diéguez-Uribeondo, J. (2014). Global distribution of two fungal pathogens threatening endangered sea turtles. PLoS ONE, 9(1), e85853. https://doi.org/10.1371/journal.pone.0085853

Solomon, S. E., and Tippett, R. (1987). The intra-clutch localisation of fungal hyphae in the eggshells of the leatherback turtle (Dermochelys coriacea ). Animal Technology 38, 73-79

Smyth, C. W., Sarmiento-Ramírez, J. M., Short, D. P. G., Diéguez-Uribeondo, J., O’Donnell, K., \& Geiser, D. M. (2019). Unraveling the ecology and epidemiology of an emerging fungal disease, sea turtle egg fusariosis (STEF). PLOS Pathogens, 15(5), e1007682. https://doi.org/10.1371/journal.ppat.1007682

Wyneken, J., Burke, T. J., Salmon, M., and Pederson, D. K. (1988). Egg failure in natural and relocated sea turtle nests. Journal of Herpetology 22, 88-96.

Figures 
a.

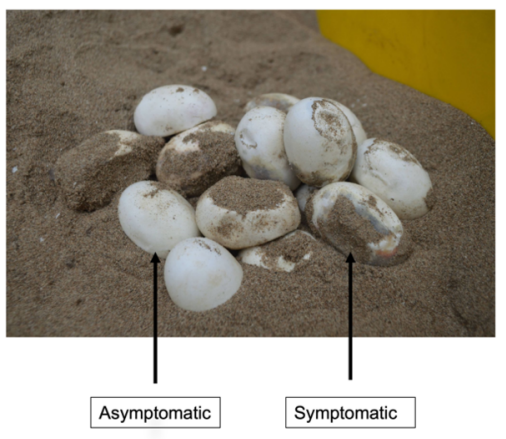

b.

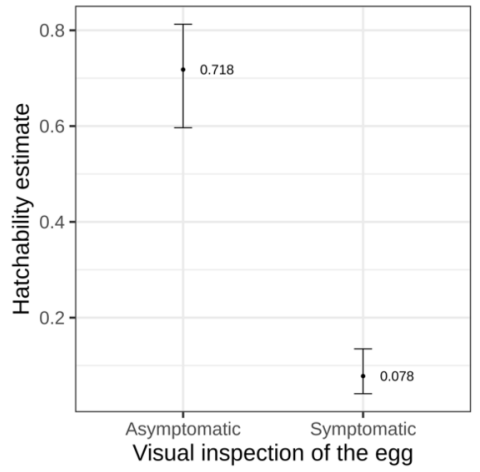

Figure 1. Illustration and hatching success of asymptomatic and symptomatic turtle eggs. a) Picture showing eggs of the yellow-spotted freshwater turtle which are asymptomatic and symptomatic of fusariosis; b) Hatchability estimates and associated $95 \%$ confidence intervals according to visual inspection of eggs (GLMM fitted with binomial distribution with nest ID as random factor).

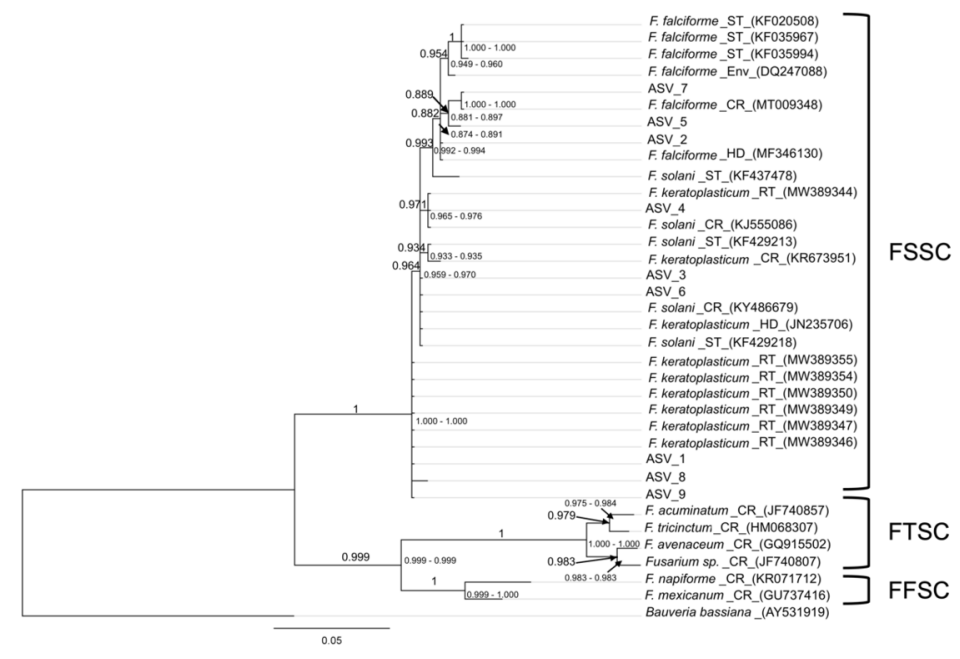

Figure 2. Bayesian phylogenetic tree of the Fusariumspp. based on sequence information of the TEF1[?] gene. ASV 1 to ASV 9 were isolated from the yellow-spotted Amazon river turtle (present study) and visualized together with Fusarium spp. isolated from different sources $(\mathrm{CR}=$ isolates from crops; $\mathrm{HD}=$ isolates from species causing diseases in humans; Env= isolates from the environment $\mathrm{RT}=$ isolates from the yellow-spotted river turtle and ST= isolates from sea turtle eggs). Strains are grouped according to the corresponding Fusarium complexes: Fusarium solani species complex (FSSC), Fusarium tricinctum Species Complex (FTSC) andFusarium fujikuroi Species Complex (FFSC). The tree was rooted with a TEF-1[?] sequence from Beuveria bassiana (AY531919). The branch labels show the posterior probability values (PP) and node labels show the $95 \%$ confidence intervals based on the probability range. 
a.

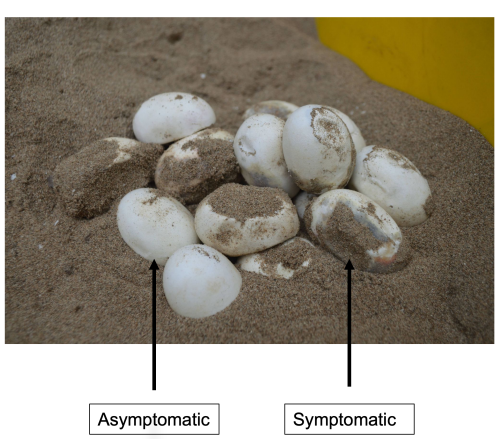

b.

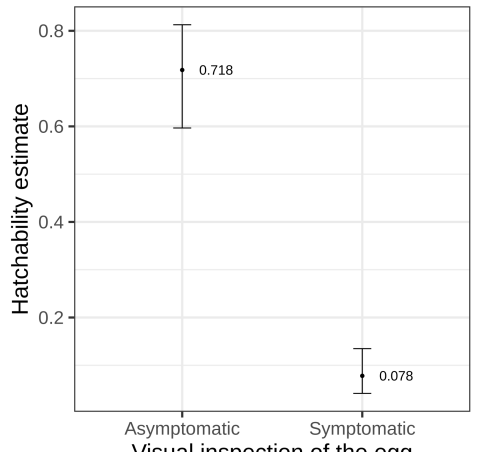

Visual inspection of the egg

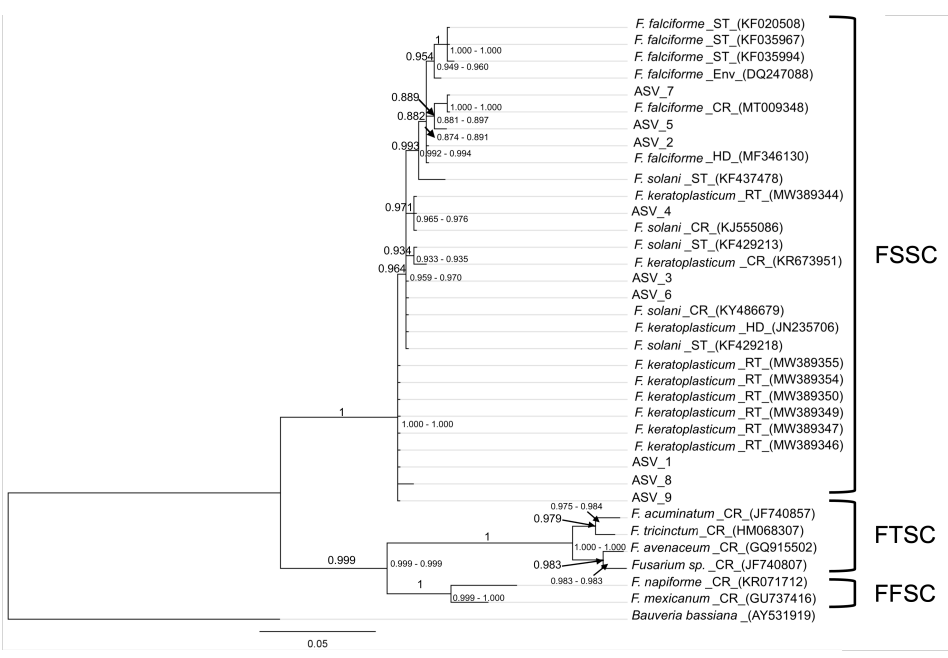

